\title{
Wavelength Tuning of a Solid-State Laser with a Tilting MEMS Micromirror
}

\author{
Alan Paterson, Ralf Bauer, Ran Li, Walter Lubeigt, Deepak Uttamchandani \\ University of Strathclyde, Centre for Microsystems and Photonics \\ Glasgow, UK
}

\begin{abstract}
Wavelength tuning of a Yb:KGW solidstate laser is presented using an electrothermallyactuated micromirror and a diffraction grating or dispersing prism. $27 \mathrm{~nm}$ and $7.5 \mathrm{~nm}$ tuning ranges are achieved using extracavity and intracavity configurations respectively.
\end{abstract}

Keywords-MEMS, micromirror, electrothermal actuation, solid-state laser, wavelength tuning

\section{INTRODUCTION}

Use of optical MEMS in lasers has been an important area of optical MEMS applications in recent years. Being compact, low-power and low-cost with batch fabrication makes MEMS ideal candidates for replacing bulk optics with similar functionalities. Added functionality has been investigated in solidstate lasers through the use of adaptive optics [1] and MEMS for active laser Q-switching [2-4]. Qswitching with MEMS has also been demonstrated in fiber lasers [5] and a microchip laser [6]. Spatial and temporal control of solid-state laser outputs have therefore been demonstrated using intracavity MEMS.

This work presents spectral control of solid-state laser output properties using a MEMS micromirror combined with wavelength dispersing or diffracting optics. Although demonstrated previously in vertical cavity surface emitting lasers and DFB lasers [7], the concept of MEMS facilitated wavelength tuning is yet to be fully investigated in high-power solid-state lasers. We present the use of a tilting micromirror and a diffraction grating or dispersing prism in a Littman configuration to actively tune the output wavelength of a Yb:KGW laser. This proof-of-concept investigation highlights the extracavity and intracavity tuning capabilities and discusses the limitations of this technique.

\section{MICROMIRROR CHARACTERIZATION}

The electrothermally actuated micromirror used in this work is shown in Figure 1. The device was fabricated using a commercially available silicon-oninsulator multi-user MEMS process offered by MEMSCAP Inc. The micromirror surface is $2 \mathrm{~mm}$ in diameter and is optically coated in a post-fabrication step with a $200 \mathrm{~nm}$ thick layer of gold, resulting in a reflectivity of $96 \%$ at $\lambda \sim 1 \mu \mathrm{m}$ and a concave surface radius of curvature (ROC) of $75 \mathrm{~mm}$. Four radially

This work was supported in part by DSTL under gran DSTLX1000090968 and by the EPSRC under grant EP/L505080/1

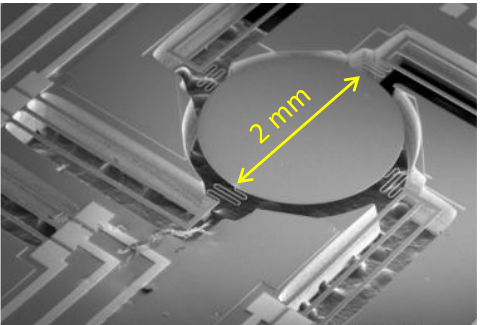

Figure 1: Scanning electron microscope image of the micromirror

positioned actuators consist each of three longitudinal beams of length $1800 \mu \mathrm{m}$, width $50 \mu \mathrm{m}$ and lateral gaps of $150 \mu \mathrm{m}$. They are connected at one end by a $60 \mu \mathrm{m}$ wide beam which also connects the actuator to the micromirror via a serpentine spring.

Actuation is achieved by passing a current through the two outer beams of the actuator, causing Joule heating and thermal expansion. The central beam is electrically isolated, acting as a constraint to force an out-of-plane movement of the actuator and hence tilt the micromirror. The positioning of the actuators enables 2D static control of the micromirror tilt angle. An $11 \mathrm{~V}_{\mathrm{dc}}$ signal applied to an actuator results in a micromirror tilt angle of $1.2^{\circ}$, which is the maximum voltage applied to prevent thermal damage to the actuators. The minimum threshold for movement was measured to be $4 \mathrm{~V}_{\mathrm{dc}}$.

\section{EXPERIMENTAL LASER RESULTS}

\section{A. Extracavity Wavelength Tuning}

A $10 \times 5 \times 2 \mathrm{~mm} \mathrm{Yb:KGW} \mathrm{crystal} \mathrm{was} \mathrm{end-pumped}$ by a fiber-coupled diode laser at $\lambda=981 \mathrm{~nm}$. Yb:KGW was used for its large gain bandwidth, enabling theoretical lasing between $1020 \mathrm{~nm}$ and $1060 \mathrm{~nm}$. A two mirror laser cavity was aligned around the crystal, consisting of a high reflection (HR) mirror with $\mathrm{ROC}=250 \mathrm{~mm}$ and a flat, $80 \%$ reflective mirror as the output coupler. This setup is shown in Figure 2, together with the extracavity feedback configuration used to enable tuning of the laser output wavelength. The feedback configuration consists of a HR folding mirror with $\mathrm{ROC}=250 \mathrm{~mm}$, a Thorlabs GR25-0310 diffraction grating and the micromirror. Laser output occurs from the $0^{\text {th }}$ order reflection of the grating. The micromirror tilt angle selects the feedback wavelength diffracted by the grating. 


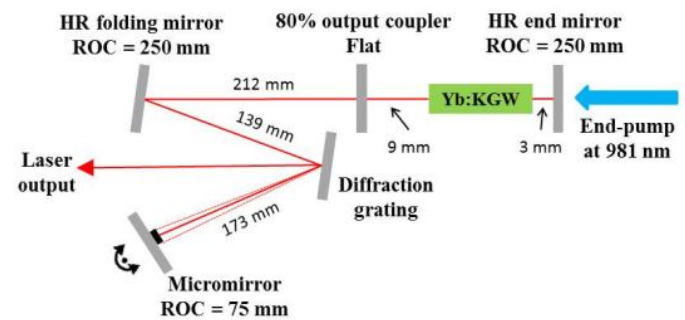

Figure 2: Extracavity laser configuration with a micromirror and a grating

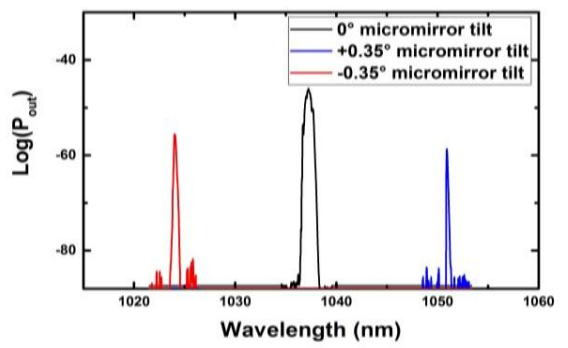

Figure 3: Plot of laser output wavelength as the micromirror is tilted for the laser in Figure 2

With no micromirror actuation the laser output had a peak wavelength of $1037 \mathrm{~nm}$, a full width half maximum (FWHM) linewidth of $0.6 \mathrm{~nm}$ and an output power of $17 \mathrm{~mW}$. By changing the micromirror tilt angle by $0.35^{\circ}$ in either direction $\left(7.2 \mathrm{~V}_{\mathrm{dc}}\right.$ applied to opposite actuators sequentially) the laser output wavelength could be tuned from $1024 \mathrm{~nm}$ to $1051 \mathrm{~nm}$, as shown in Figure 3. The FWHM linewidths were $0.2 \mathrm{~nm}$ and $0.1 \mathrm{~nm}$ respectively and the laser output powers were $6 \mathrm{~mW}$ and $2.5 \mathrm{~mW}$ respectively.

\section{B. Intracavity Wavelength Tuning}

Due to the losses induced by the grating, a fully intracavity configuration was not possible. Therefore, another laser cavity was constructed using a prism to disperse the available incident wavelengths. The HR end-mirror, with $\mathrm{ROC}=250 \mathrm{~mm}$, of the previous cavity was retained with the output side now consisting of an output coupler with $\mathrm{ROC}=250 \mathrm{~mm}$ and $98 \%$ reflectivity, a dispersing prism and the micromirror. The prism was angled close to the Brewster angle for the incident laser light. The cavity configuration is shown in Figure 4.

With no micromirror actuation the laser output had a peak wavelength of $1027 \mathrm{~nm}$ with a FWHM linewidth of $0.4 \mathrm{~nm}$ and an output power of $25 \mathrm{~mW}$. For micromirror tilt angles of $0.1^{\circ}$ in either direction, the wavelength was tunable between $1024 \mathrm{~nm}$ and $1031.5 \mathrm{~nm}$, as shown in Figure 5, with FWHM linewidths of $0.4 \mathrm{~nm}$ and $0.2 \mathrm{~nm}$ respectively and output powers of $20 \mathrm{~mW}$ and $4 \mathrm{~mW}$ respectively.

\section{CONCLUSION}

Wavelength tuning of a solid-state laser with a micromirror has been demonstrated with extracavity and intracavity configurations. A diffraction grating and a micromirror in an extracavity configuration

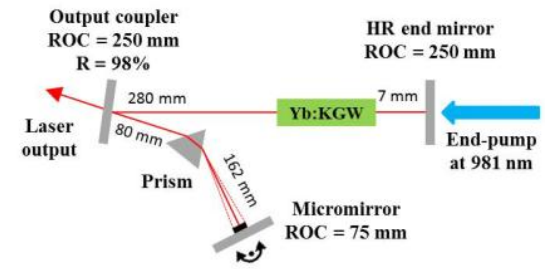

Figure 4: Intracavity laser configuration with a micromirror and a prism

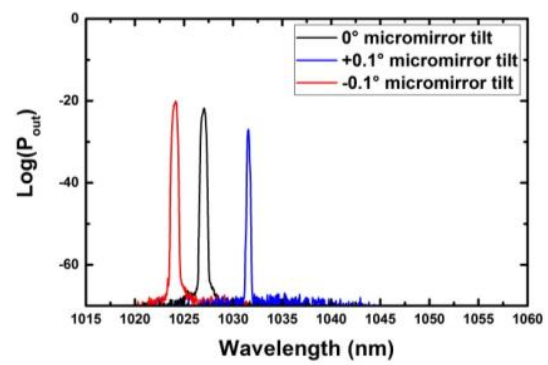

Figure 5: Plot of laser output wavelength as the micromirror is tilted for the laser in Figure 3

resulted in a wavelength tuning range of $27 \mathrm{~nm}$. With a dispersing prism and a micromirror in an intracavity configuration the wavelength was tunable by $7.5 \mathrm{~nm}$. Future work will be focused on optimizing the technique. A particular area of interest is improvement to the MEMS optical coating which is the current limiting factor in power-scaling the laser and the reason for the proof-of-principle demonstration being in the $\mathrm{mW}$ range. A low-stress dielectric coating would allow higher laser output powers suitable for defense and industrial applications.

\section{REFERENCES}

[1] W. Lubeigt, G. Valentine, J. Girkin. E. Bente and D. Burns, "Active transverse mode control and optimisation of an allsolid-state laser using an intracavity adaptive-optic mirror," Opt. Exp., vol. 10, pp. 550-555, July 2002.

[2] R. Bauer, A. Paterson, C. Clark, W. Lubeigt and D. Uttamchandani, "Output characteristics of Q-switched solidstate lasers using intracavity MEMS micromirrors," IEEE JSTQE, vol. 21, pp. 356-363, August 2014.

[3] R. Bauer, W. Lubeigt and D. Uttamchandani, "Dual Qswitched laser outputs from a single lasing medium using an intracavity MEMS micromirror array," Opt. Let., vol. 37, pp. 3567-3569, Sept 2012.

[4] V. Milanović et al., "Compact MEMS mirror based Q-switch module for pulse-on-demand laser range finders," SPIE MOEMS and Min. Sys. XIV, Feb 2015.

[5] M. Fabert, A. Desfarges-Berthelemot, V. Kermène and A. Crunteanu, "Temporal synchronization and spectral combining of pulses from fiber lasers Q-switched by independent MEMS micro-mirrors," Optics Express, Vol. 20, pp. 22895-22901, Sept 2012.

[6] A. Inoue, T. Komikado, K. Kinoshita, J. Hayashi and S. Umegaki, "Deformable mirror for mechanical Q-switching of laser-diode-pumped microchip laser,” Jap. Jour. Appl. Phys., Vol. 46, pp. L1016-L1018, October 2007.

[7] A. Liu and M. Zhang, "A review of MEMS external-cavity tunable lasers," J. Micromech. And Microeng., Vol. 17, pp. 